\title{
Effect of the bonding strategy on the tensile retention of full-contour zirconia crowns
}

\author{
Sabrina A. Feitosa ${ }^{\mathrm{a}, \mathrm{b}}$, Fernanda Campos ${ }^{\mathrm{b}}$, Walter Kenji Yoshito ${ }^{c}$, Dolores R.R. Lazar ${ }^{\mathrm{c}}$, \\ Valter Ussui $^{\mathrm{c}}$, Luiz Felipe Valandro ${ }^{\mathrm{b}, \mathrm{d}}$, Marco A. Bottino ${ }^{\mathrm{b}}$, Marco C. Bottino ${ }^{\mathrm{e}, *}$

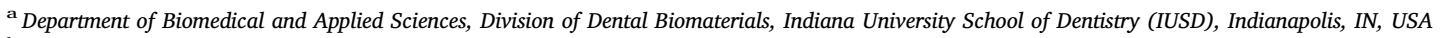 \\ ${ }^{\mathrm{b}}$ Department of Dental Materials and Prosthodontics, Institute of Science and Technology, Universidade Estadual Paulista-UNESP, São José dos Campos, SP, Brazil \\ ${ }^{\mathrm{c}}$ Materials Science and Technology Center, Institute for Energy and Nuclear Research (IPEN), São Paulo, SP, Brazil \\ d PhD Graduate Program in Oral Sciences (Prosthodontics Unit), School of Dentistry, Federal University of Santa Maria, Santa Maria, RS, Brazil \\ e Department of Cariology, Restorative Sciences, and Endodontics, University of Michigan School of Dentistry, 1011N. University Ave (Rm 5223), Ann Arbor, MI, USA
}

A R T I C L E I N F O

\section{Keywords:}

Adhesion

Zirconia

Surface conditioning

Properties

Yttria-stabilized tetragonal zirconia

polycrystals

MDP

Retention strength

\begin{abstract}
A B S T R A C T
This study evaluated the effect of distinct bonding strategies on the retention of full-contour zirconia ceramic (YTZP, FCZ) crowns, and it characterized some physicochemical and mechanical properties of FCZ ceramic and its corresponding glazing system. To evaluate retention strength, dies were made with a dentin-analogue material to simulate a prepared tooth. FCZ crowns were manufactured using CAD-CAM technology and allocated into groups according to the bonding strategy: no ceramic treatment (PF - Panavia F cementation), glaze (GL), tribochemical silica coating (CJ), CJ + GL, and piranha solution followed by glaze (PS + GL). The specimens were subjected to thermocycling and storage in distilled water for 100 days before the retention tests. FCZ presented a porosity volume fraction of $0.2 \%$, an apparent density of $6.06 \mathrm{~g} / \mathrm{cm}^{3}$, Vickers hardness of $12.4 \pm 0.07 \mathrm{GPa}$, and fracture toughness of $5.54 \pm 0.24 \mathrm{MPa} \mathrm{m}^{1 / 2}$. SEM revealed a homogeneous microstructure composed of submicron-sized grains. XRD identified mainly zirconia's tetragonal phase. Glaze powder morphology was observed to be irregular, with a nanometric particle size, and a diffraction pattern characteristic of an amorphous material with several peaks of leucite. The PF and GL groups had higher retention values. The majority of the groups presented pre-test bonding failures, and two catastrophic failures of the FCZ-crown (GL and PF groups) were noted. The use of an MDP-containing resin cement or glaze application might improve retention of the FCZ crowns.
\end{abstract}

\section{Introduction}

Zirconia has gained increased attention as a dental restorative material due to its proven biocompatibility, mechanical strength, and its use in Computer-Aided Design/Computer-Aided Machining (CAD/ CAM) technology [1-3]. Superior mechanical properties, particularly those as a consequence of the transformation-toughening mechanism, have also contributed to zirconia's popularity. Induced by mechanical stress, the phase transformation from tetragonal to monoclinic $(\mathrm{t} \rightarrow \mathrm{m})$ produces an increased grain volume of 3-4\% [1-3]. The restricted volume expansion results in the development of compressive stresses, which oppose crack propagation within the ceramic and along its surface [1-3]. The transformation-toughening mechanism can be controlled by adding stabilizing oxides or dopants, such as yttrium oxide, thus yielding the dental zirconia most commonly used-3-mol\% yttriastabilized tetragonal zirconia polycrystals (3Y-TZP) [1-3].
Clinically, 3Y-TZP ceramic is employed as the core material for fixed partial dentures (FPDs) or single crowns, with a veneering ceramic layered or pressed onto the occlusal surface. However, clinical studies continue to report failures, such as debonding, chipping, or fracture of the veneering ceramic [4-7]. These failures can be attributed to multiple factors, such as the differences in material properties (e.g., the coefficient of thermal expansion [CTE] between the zirconia core and the veneering ceramic), the cooling rate and geometry of the bilayer ceramic structure, and difficulty in promoting a strong, reliable bond between the zirconia surface and tooth structure. To prevent such failures, full-contour 3Y-TZP zirconia crowns (FCZ) without veneering porcelain have been developed [8]. These are specifically indicated for the posterior areas of the mouth, where esthetics are not the primary focus and for cases with limited occlusal and palatal space $[9,10]$.

Despite significant improvement in bonding between zirconia and resin cement, it is difficult to precisely predict clinical outcomes,

\footnotetext{
* Corresponding author.

E-mail address: mbottino@umich.edu (M.C. Bottino).
} 
because more frequently, the bond is compromised if its internal (intaglio) surface is not properly treated. Zirconia has a high crystalline content that makes hydrofluoric acid (HF) unable to effectively attack its surface [11,12]. Several studies have investigated alternative bonding strategies to improve the bond strength of resin cements to zirconia [11-19]. Particularly, studies have focused on surface treatments using airborne particle abrasion with aluminum oxide $\left(\mathrm{Al}_{2} \mathrm{O}_{3}\right)$ or aluminum oxide particles modified by silica. However, the effects of airborne particle abrasion can affect the mechanical properties of zirconia, resulting in phase transformation $(\mathrm{t} \rightarrow \mathrm{m})$ and long-term deleterious effects $[20,21]$. To avoid side effects from particle abrasion, studies have investigated the application of a thin layer of a vitreous material (i.e., glaze) on the zirconia internal surface [13-16]. Glaze is a vitreous material, sensitive to $\mathrm{HF}$ etching, which can make the zirconia surface chemically reactive and mechanically retentive [15]. An alternative approach would be a chemical treatment using the piranha solution (PS), which is a mixture of sulfuric acid and hydrogen peroxide (3:1), known as a strong oxidizing solution that not only makes most surfaces hydrophilic by hydroxylation, but also improves the hydroxylation and bond strength of adhesive monomers [17].

Therefore, the aims of this investigation were to: 1) characterize some physicochemical and mechanical properties of FCZ ceramic; 2) characterize its corresponding glaze, which needs to be considered when working with monolithic zirconia restorations; and 3) evaluate the effects of distinct bonding strategies on the retention of FCZ crowns.

\section{Materials and methods}

\subsection{FCZ preparation}

FCZ ceramic bars (3Y-TZP, Lot\#P02286, Diazir ${ }^{\circledR}$, Ivoclar-Vivadent, Amherst, NY, USA) were cut into blocks $\left(10 \times 10 \times 3 \mathrm{~mm}^{3}\right)$ using a diamond blade mounted on a precision saw machine (ISOMET 1000, Buehler Ltd., Lake Bluff, IL, USA) [2]. The ceramic blocks were sintered at $1500^{\circ} \mathrm{C}$ for $2 \mathrm{~h}$ in a high-temperature furnace (Lindberg Blue $\mathrm{M}$, Asheville, NC, USA). Samples were polished with 9, 6, 3, and $1 \mu \mathrm{m}$ ceramographic cloth and diamond pastes (Préparations Diamantées Mecaprex, Grenoble, France). All samples were immersed in isopropyl alcohol and cleaned for $5 \mathrm{~min}$ in an ultrasonic bath (Vitasonic, Vita Zahnfabrik, Bad Säckingen, Germany) [2].

\subsection{FCZ microstructural characterization}

Density and porosity of the sintered ceramics were determined using an immersion method based on the Archimedes principle [2]. The polished sintered ceramic blocks received a thermal treatment in order to reverse the phase transformation $(\mathrm{m} \rightarrow \mathrm{t})$ produced during ceramographic procedures and reveal the post-sintering microstructure using a scanning electron microscope (SEM, XL30, Phillips, Eindhoven, The Netherlands) [2]. The zirconia grain diameter was measured using the ImageJ software (National Institutes of Health, Bethesda, MD, USA). Xray diffraction (XRD) (DMAX 2000, model Multiflex, Rigaku Corporation, Tokyo, Japan) analysis was performed to identify the crystalline phase (tetragonal/cubic/monoclinic) by using $\mathrm{Cu} / \mathrm{Ka}$ radiation with a scan range of $5-90^{\circ}$, step of $0.02^{\circ}$, and a counting time of $8 \mathrm{~s} / \mathrm{step}$.

\subsection{FCZ mechanical test}

Hardness was determined by the Vickers hardness test (VMT-7, Buehler). Briefly, 3 samples were embedded in bakelite and polished with a ceramographic cloth and diamond suspensions (Préparations Diamantées Mecaprex). A $10 \mathrm{kgf}$ load was applied for $15 \mathrm{~s}$ by the indenter, and the impressions were taken from 6 areas per specimen. The values were calculated using Eq. (1), where "P" is the applied load $(\mathrm{N})$, "d" is the diagonal length (m), and "a" is the angle between the opposite faces of the indenter $\left(136^{\circ}\right)[2,20]$.
$H v=\frac{\propto P}{d^{2}}$

Fracture toughness $\left(\mathrm{K}_{\mathrm{IC}}\right)$ was calculated by observing crack type, dimensions of the indentations, and cracks on the ceramics during indentation using an optical microscope (PMG3, Olympus, Tokyo, Japan). The crack type was identified by investigating the indented surface polished with a diamond paste, following previously established criteria and equations $[21,22]$.

\subsection{FCZ glaze preparation}

Diazir ${ }^{\varpi}$ full-contour glaze paste (Lot\#P4VM, Ivoclar-Vivadent) was dried in an Electric Muffler Oven (model 1857, Fornitec Indústria e Comércio Ltda, São Paulo, Brazil) at $200^{\circ} \mathrm{C}$ to obtain the glaze powder.

\subsection{Glaze morphological and chemical characterizations}

Powder particle size distribution was evaluated using Dynamic Light Scattering (DLS, Brookhaven Instruments Corporation, Holtsville, NY, USA) and the data were analyzed using dedicated software (ZetaPlus Particle Sizing Software Ver.4.02, Brookhaven Instruments Corporation). The glaze mineral phase identification was performed by $\mathrm{X}$-ray diffraction. The diffraction pattern was interpreted by comparing it with the ICDD (International Centre for Diffraction Data, Newtown, PA, USA) card files. Morphology of the obtained glaze powder was carried out via SEM (Tabletop, Model TM 3000, Hitachi HighTechnologies, Krefeld, Germany).

\subsection{Epoxy resin dies preparation}

Epoxy resin (NEMA Grade G10 glass-reinforced epoxy plastic laminates, Accurate Plastics, Inc., Yonkers, New York, USA) dies were prepared (Centro para Inovação e Competitividade do Cone Leste Paulista, Parque Tecnológico de São José dos Campos, SP, Brazil) to simulate a single-tooth FCZ crown, i.e., a base of $8 \mathrm{~mm}$ (diameter) $\times$ $6 \mathrm{~mm}$ (height), preparation of $6 \mathrm{~mm}$ in height, rounded shoulder finish line, and $20^{\circ}$ of total occlusal convergence angle [23].

\subsection{FCZ crown preparation}

FCZ disks were cut into blocks using a diamond blade mounted on a precision saw machine (ISOMET 1000, Buehler). The blocks presented the same dimensions as the commercial blocks for the CAD-CAM system IPS e.max ${ }^{\oplus}$ ZirCAD, (MO C15L, Ivoclar-Vivadent). All the blocks were finished with $\mathrm{SiC}$ papers under water-cooling to standardize the surfaces. A milling mandrel was glued to each FCZ block with cyanoacrylate gel, resulting in a CAD-CAM block that could be attached to the milling unit. FCZ crowns were milled with retentions (Fig. 1) on the occlusal surface $[18,19,24]$ using the inLab MC XL Milling Unit (Sirona Dental Systems, Bensheim, Germany) and designated software (Inlab 3.60). After milling, all crowns were washed with distilled water, dried in an oven (Pyro-oven, H.D. Justi Company, Philadelphia, PA, USA), and sintered (Programat S1, Ivoclar-Vivadent) following the manufacturer's instructions.

\subsection{Bonding strategy}

Prior to the bonding protocol, the FCZ crowns were cleaned in alcohol in an ultrasonic bath for $5 \mathrm{~min}$, rinsed in DI water, and dried with oil-free canned air. The samples were allocated into 5 groups $(\mathrm{N}=12$ / group) based on the bonding strategy (Table 1): CJ, GL, PF, CJ + GL, and PS + GL. For surface pretreatment, G10 dies were etched with HF (Lot\# R53559, Ivoclar-Vivadent) for $60 \mathrm{~s}$, and a thin layer of a silane coupling agent (Lot\# R50513, Monobond Plus, Ivoclar-Vivadent) was applied [23]. 

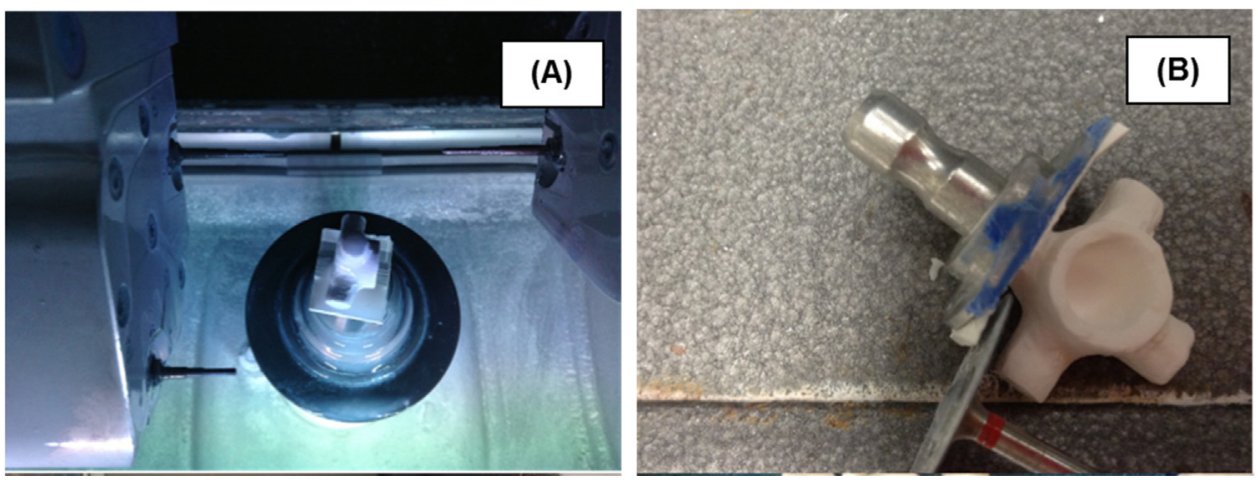

Fig. 1. Fabrication of monolithic zirconia crowns for the tensile retention test. (A) Modified monolithic zirconia crown obtained from a CAD-CAM zirconia block attached to a CAD-CAM machine. (B) An FCZ crown before sintering in a dedicated furnace for zirconia.

The glazed-treated crowns (groups: GL, CJ + GL, and PS + GL) were etched with $5 \% \mathrm{HF}$ for $90 \mathrm{~s}$, silanized for $60 \mathrm{~s}$, gently air-dried for $5 \mathrm{~s}$, and bonded using a 10-methacryloyloxydecyl dihydrogen phosphate/MDP-free resin cement (Multilink ${ }^{\star}$ Automix, Lot\# S35929, Ivoclar-Vivadent) according to the manufacturer's instructions. The PF group (no surface pretreatment) was bonded with an MDP-containing resin cement, Panavia F (Lot\# 051234, Kuraray Noritake Dental Inc., Okayama, Japan), following the manufacturer's instructions. The crowns were cemented using a device that applied a force of $750 \mathrm{~g}$ $[18,19]$ over the crown/G10 to standardize the resin cement layer and light-cured (Radii-Cal, SDI, Melbourne, Victoria, Australia) for $40 \mathrm{~s}$ in 4 different areas (corresponding with each crown retention). After the bonding protocol, the samples were stored in distilled water at $37^{\circ} \mathrm{C}$ for $24 \mathrm{~h}$. All the samples were then subjected to thermocycling (TC) $\left(6000 \times\right.$ cycles, $\left.5^{\circ} \mathrm{C}-55^{\circ} \mathrm{C}\right)$ and stored at $37^{\circ} \mathrm{C}$ in distilled water for 100 days.

\subsection{Tensile retention strength test}

After themocycling/storage, the crown was embedded in acrylic resin until it was covered by the ceramic retentions and the die base was also embedded in acrylic resin. Care was taken to protect the adhesive FCZ/G10 interface of the acrylic resin. The FCZ/G10 samples were embedded in acrylic resin using a dental surveyor to keep the long axis of the sample perpendicular to the ground plane. Then, samples were submitted to a tensile retention test using a universal testing machine (Emic, DL-1000, São José dos Pinhais, Brazil). The crown was connected to a mobile device that contained a load cell of $1000 \mathrm{~N}$ on the upper side. The tensile retention test was performed at a crosshead speed of $0.5 \mathrm{~mm} / \mathrm{min}[18,19]$. The tested surfaces were analyzed under a stereomicroscope (steREO Discovery-V20, Carl Zeiss, Göttingen, Germany). The type of failure was classified based on the conditions defined in Fig. 2. Representative samples were carefully sectioned using a diamond blade, sputter-coated with $\mathrm{Au}$, and analyzed using SEM (Inspect S50, FEI Netherlands, Eindhoven, The Netherlands).

\subsection{Statistical analysis}

The tensile retention strength (kgf) data were statistically analyzed using the non-parametrical test, Kruskal-Wallis, followed by the Dunn post-hoc test, to determine significant differences among the bonding strategies. A 5\% significance level was used to analyze the data.

\section{Results}

\subsection{FCZ and glaze characterizations}

SEM imaging (Fig. 3A) showed spherical grain surfaces with homogeneous distribution, well-defined boundaries without pores, and a mean grain diameter of $0.75 \pm 0.2 \mu \mathrm{m}$. Further analysis of the crystalline structure was performed by XRD and the data was interpreted using ICDD cards: file 89-7710 for the tetragonal phase and file 81-1551 for the cubic phase. Results of the XRD analysis revealed a tetragonal phase structure profile for the FCZ crystals. Then, Rietveld analysis was used to refine the crystalline structure. This method used the file 99022 for the tetragonal phase, and file 72956 for the cubic phase (ICSD, Inorganic Crystal Structure Database).

FCZ exhibited a porosity volume fraction of ca. $0.2 \%$ and an apparent density of $6.06 \mathrm{~g} / \mathrm{cm}^{3}$, which is approximately $99.34 \%$ of the theoretical density value $\left(\mathrm{p}=6.10 \mathrm{~g} / \mathrm{cm}^{3}\right)$.

The Vickers hardness value of FCZ zirconia was $12.4 \pm 0.07 \mathrm{GPa}$. After examination of the polished, indented surface (Fig. 3B), fracture toughness was determined using Palmqvist's crack formation model proposed by Ponton and Rawlings. Fracture toughness of the FCZ was found to be $5.54 \pm 0.24 \mathrm{MPa} \mathrm{m}^{1 / 2}$.

Morphology of the FCZ glaze powder was observed to be irregular in

Table 1

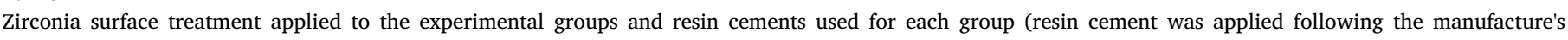
instruction).

\begin{tabular}{|c|c|c|}
\hline Groups & Zirconia surface treatment & Resin cement \\
\hline GL $^{*}$ & $\begin{array}{l}\text { A thin layer of glaze (Diazir full-contour glaze paste, Lot\# P4VM, Ivoclar-Vivadent) was applied on the inner surface of the zirconia crowns } \\
\text { using a microbrush. The glaze liquid excess was dried. The glazed crown was sintered following the manufacturer's instructions. }\end{array}$ & Multilink automix \\
\hline PF & Without zirconia surface pretreatment & Panavia F \\
\hline $\mathbf{C J}+\mathbf{G L}$ & $\begin{array}{l}\left.\text { Zirconia crowns were submitted to particle abrasion using CoJet }{ }^{\circ} \text {-Sand ( } 30 \mu \mathrm{m} \text { - CoJet }{ }^{\circ} \text {-Sand, } 3 \mathrm{M} \text { ESPE AG, Seefeld, Germany; Lot\# } 501661\right) \text { for } \\
30 \mathrm{~s}(\sim 10 \mathrm{~mm} \text { distance, } 2.8 \text { bar) followed by the glaze application. The glaze liquid excess was dried. The glazed crown was sintered following } \\
\text { the manufacture's instructions. }\end{array}$ & Multilink automix \\
\hline $\mathbf{P S}+\mathbf{G L}^{*}$ & $\begin{array}{l}\text { Zirconia crowns were submitted to a chemicall treatment using piranha solution on a 3:1 ratio - Sulfuric acid }\left(\mathrm{H}_{2} \mathrm{SO}_{4}\right) / 30 \% \mathrm{H}_{2} \mathrm{O}_{2} \text {, rinsed with } \\
\text { distilled water form } 5 \text { min and kept into the becker with distilled water for } 20 \mathrm{~min} \text {. After drying, the crowns received a thin layer of glaze into the } \\
\text { inner surface. The glaze liquid excess was dried. The glazed crown was sintered following the manufacture's instructions. }\end{array}$ & Multilink automix \\
\hline CJ & Zirconia crowns were submitted to particle abrasion using CoJet ${ }^{\circ}$-Sand during $30 \mathrm{~s}$. & Multilink automix \\
\hline
\end{tabular}

* FCZ glaze (GL) was manipulated always the same ration glaze / correspondent glaze liquid. 


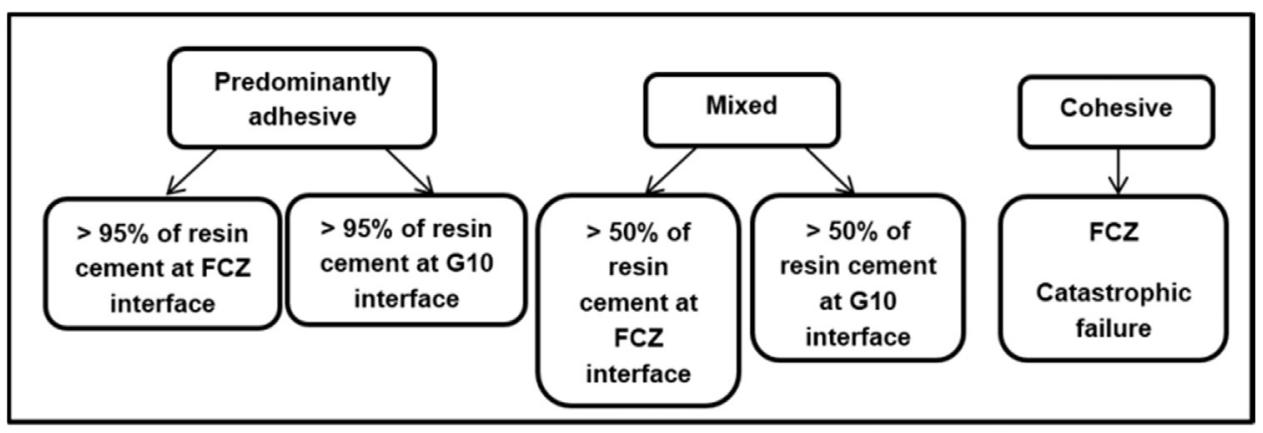

Fig. 2. Classification used to determine the type of failure in the samples subjected to the tensile retention strength test.

shape and size, with an average particle size distribution of $148.51 \mathrm{~nm}$ (Fig. 4A). Mineral phase identification of the powder was performed using XRD by comparing the resulting pattern's peak intensities to the ICDD file 01-085-1626. The analyzed powder presented a diffraction pattern characteristic of an amorphous material with several peaks of leucite (Fig. 4B).

\subsection{Tensile retention strength test}

Pretest failures were observed in all groups except PF. Catastrophic failure of the FCZ crown was observed in the GL and PF groups. Groups GL $(\sim 25 \%)$, CJ + GL $(\sim 17 \%)$, and PF $(\sim 8 \%)$ had the lowest percentage of pretest failures, while group PS + GL $(\sim 50 \%)$ had the highest. One cohesive failure of the FCZ crown was registered in each of the GL and PF groups, and corresponding to $\sim 4 \%$ of the tested samples ( $\mathrm{N}=46$, including the catastrophic failures). These crowns were evaluated using stereomicroscopy followed by SEM imaging. Small pores were observed on the FCZ fractured surface of both the GL and PF groups.

Tensile retention strength was highest in PF (47.2 kgf) (Fig. 5) and was statistically the same as in GL but higher when compared to the other groups (Fig. 5). The lowest tensile retention strength ( $6.7 \mathrm{kgf})$ was observed in the group PS + GL. In failure analysis, PF presented the highest number of mixed failures with resin cement at the G10 substrate when compared to CJ, GL, and CJ + GL (Table 2 and Fig. 6).

\section{Discussion}

In this study, we manufactured FCZ crowns using CAD-CAM technology and subjected them to various bonding strategies in an attempt to improve retention strength. Prior to subjecting the FCZ crowns to bonding strategies, we analyzed selected physicochemical and mechanical properties. The FCZ ceramic manufactured in this study presented a porosity volume fraction of $0.2 \%$ and an apparent density of $6.06 \mathrm{~g} / \mathrm{cm}^{3}$, approximately $99.34 \%$ of the theoretical value $(\mathrm{p}=$ $6.10 \mathrm{~g} / \mathrm{cm}^{3}$ ). These values are similar to those previously reported in the literature $[2,3]$. Although grain size of the FCZ was larger than the expected $0.3 \mu \mathrm{m}^{2}$ (actual particle size was $0.75 \mu \mathrm{m}$ ), the porosity and density of the ceramic were not negatively affected.

Indentation hardness is the simplest technique available to evaluate the mechanical properties of a material. Vickers hardness values of $10.3 \pm 0.2 \mathrm{GPa}, 10.6 \pm 0.4 \mathrm{GPa}, 18.4 \pm 0.5 \mathrm{GPa}$, and $13.5 \pm 0.2 \mathrm{GPa}$ have been reported for In-Ceram Zirconia Block, In-Ceram Zirconia, Procera All Ceram, and an experimental 3Y-TZP zirconia processed by co-precipitated 3Y-TZP nanosized powders, respectively [2]. In this study, the calculated Vickers hardness value of FCZ was $12.4 \pm 0.07 \mathrm{GPa}$, which is well within the range of the reported values. After examination of the polished indented surface, fracture toughness was measured using Palmqvist's crack formation model, because it is best suited for materials with a high fracture resistance. Fracture toughness of the FCZ investigated herein was found to be $5.54 \pm 0.24 \mathrm{MPa} \mathrm{m}^{1 / 2}$, which is similar to the values of an experimental $3 \mathrm{Y}$-TZP zirconia $\left(6.0 \pm 0.2 \mathrm{MPa} \mathrm{m}^{1 / 2}\right)$ [2].

To ensure clinical applicability of utilizing the glaze as a surface conditioning method, adhesion studies between FCZ ceramics and resin-based cements and between FCZ ceramics and their corresponding glazes, the tensile retention test was performed. The present study showed that the MDP-containing resin cement (PF) and FCZ surface pretreatment using a thin layer of glaze (GL) are potential strategies for luting zirconia restorations. The results showed no differences between GL, CJ, and the CJ + GL groups. The PF group presented the highest mean value of tensile retention strength (47.27 kgf) when compared to CJ, CJ + GL, and PS + GL. PS + GL presented the lowest values of tensile retention $(6.7 \mathrm{kgf})$. The results also suggested that FCZ

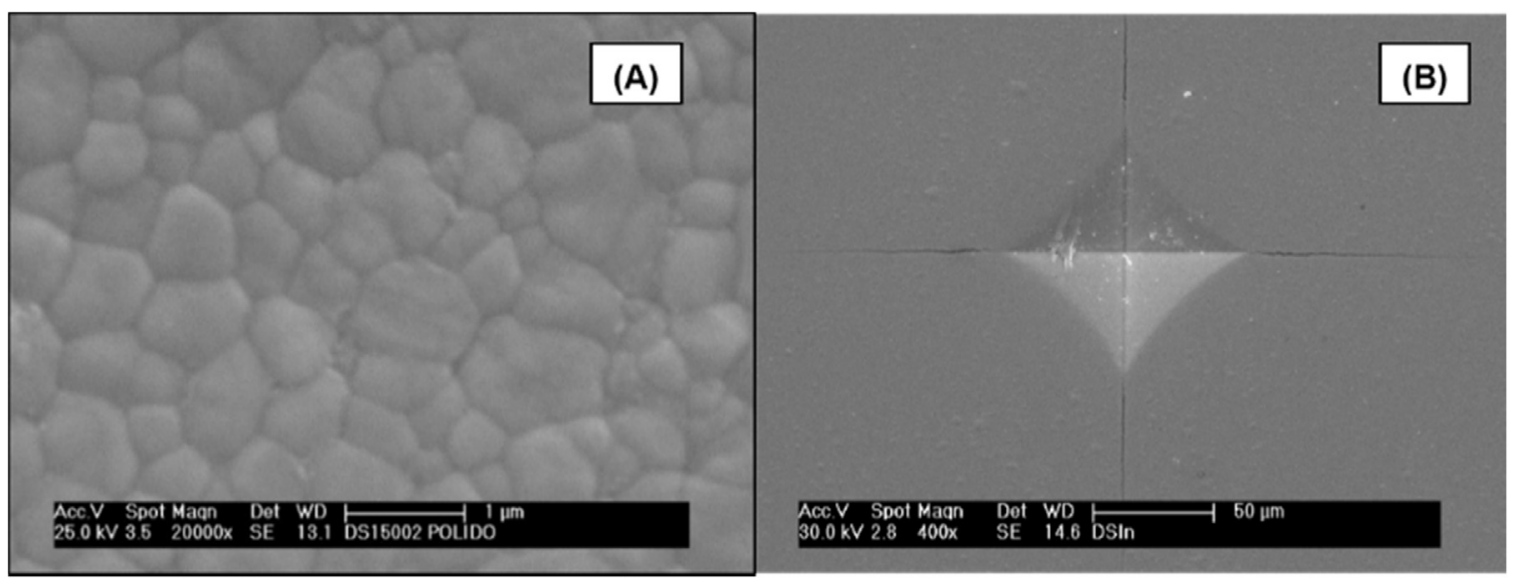

Fig. 3. (A) SEM micrograph (secondary electrons, SE mode) of the polished and thermally-etched surface of the Y-TZP ceramic sintered at $1500{ }^{\circ} \mathrm{C}$ for $2 \mathrm{~h}$. Average grain size $0.75 \pm 0.2 \mu \mathrm{m}$. (B) Representative SEM micrograph of crack formation by Vickers indentation. 

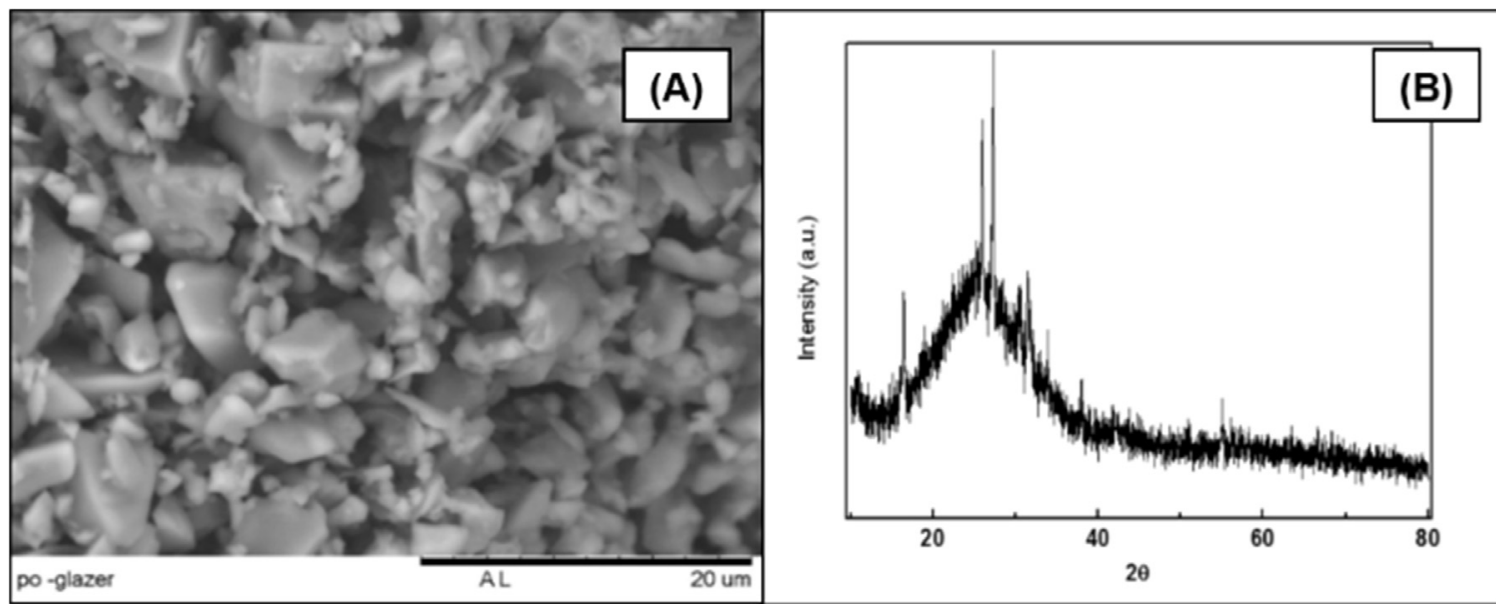

Fig. 4. (A) SEM micrograph of dried FCZ glaze powder showing irregular distribution and shape. (B) XRD pattern of the glaze: mineral identification.

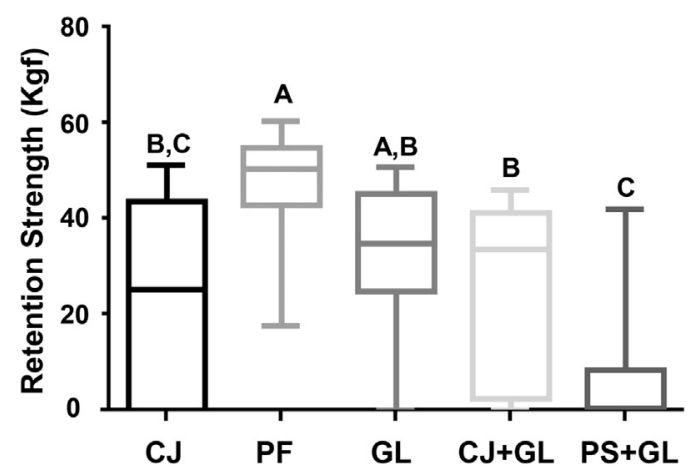

Fig. 5. Box plot of the experimental groups subjected to the tensile retention strength test. (CJ) FCZ sandblasted with aluminum oxide particles modified by silica $\mathrm{SiO}_{2}$ (Cojet ${ }^{\circ}$ ); (PF) FCZ without FCZ surface pretreatment and bonded with a MDP-containing resin cement (Panavia F); (GL) FCZ treated with a thin layer of glaze; (CJ + GL) FCZ sandblasted with aluminum oxide particles modified by silica $\mathrm{SiO}_{2}$ (Cojet ${ }^{\circ}$ ), followed by the glaze application; (PS + GL) FCZ immersed in piranha solution, immersed in distilled water (as previously mentioned), and followed by the glaze application.

pretreatments using tribochemical silica coating (CoJet $\left.{ }^{\circ}\right)$ or PS did not improve crown retention to the dentin analogue (NEMA G10). Therefore, use of a MDP-containing resin cement could result in high crown retention when compared to treatments that combine FCZ pretreatment and glaze application (CJ + GL and PS + GL). The PS + GL group treated with PS, followed by the glaze application, demonstrated decreased values in crown retention. Thus far, only a few studies have used the tensile retention test to evaluate resin bonding to zirconia crowns $[18,19,24]$. Herein, we chose G10 as a substrate in order to avoid failures, such as root fracture, and other variables related to dentin structure (position or number of dentinal tubules) and tooth preparation [18]. The use of the dentin analogue is justified, since it can be considered a standardized substrate in all the evaluated groups.

Previous studies have reported promising results using MDP-containing materials, including adhesives, primers, silane coupling agents, and resin cements $[12,16,25]$. Higher bond strength of MDP-containing materials can be credited to the chemical interaction between zirconia and MDP [25]. The MDP functional monomer has bifunctional ends, consisting of hydrophilic ester groups that react with metal oxides, such as zirconia or alumina (inorganic substrate), and the vinyl group, which reacts with monomers in resin-based materials (organic substrate/ polymer) when polymerized [18].

In this study, chemical treatment with PS was performed to improve bond strength between FCZ + GL/resin cement and to avoid the potential negative impacts of airborne particle abrasion [26]. The results of tensile retention revealed a delicate and fragile bond strength between FCZ and G10, with lower means and highest number of pretested failures when compared to the others groups. Only a few studies have thus far used PS for zirconia surface pretreatment. A previous study reported more effective results when bonding PS-treated 3Y-TZP with Multilink (Ivoclar-Vivadent) resin cement when compared to Panavia F (Kuraray Co., Ltd., Tokyo, Japan) [17]. Another report showed that the 3Y-TZP surface treatment with particles abrasion, followed by a primer application (Alloy-Primer, Kuraray Co., Ltd.), improved bond strength to enamel when compared to the group treated only with PS [15]. The results of the present study and that of a previous study published by our group [27] support PS effectively cleaning the zirconia surface, despite its inability to create surface undercuts that, when followed by primer application, can result in a more

Table 2

Type of failure classification and (\%) of pre-test failures (PTF).

\begin{tabular}{|c|c|c|c|c|c|c|c|c|}
\hline \multirow[t]{3}{*}{ Groups } & \multicolumn{8}{|l|}{ Failures } \\
\hline & \multicolumn{2}{|l|}{ Adhesive } & \multicolumn{3}{|l|}{ Mixed } & \multirow{2}{*}{$\begin{array}{l}\text { FCZ catastrophic } \\
\text { failure }\end{array}$} & \multicolumn{2}{|c|}{$\mathbf{P T F}^{\mathrm{a}}$} \\
\hline & $\begin{array}{l}>95 \% \text { of resin } \\
\text { cement on the FCZ } \\
\text { surface }\end{array}$ & $\begin{array}{l}>95 \% \text { of resin } \\
\text { cement on the G10 } \\
\text { surface }\end{array}$ & $\begin{array}{l}>50 \% \text { of resin } \\
\text { cement on the FCZ } \\
\text { surface }\end{array}$ & $\begin{array}{l}>50 \% \text { of resin } \\
\text { cement on the G10 } \\
\text { surface }\end{array}$ & $\begin{array}{l}\sim 50 \% \text { of resin cement } \\
\text { on each substrate }\end{array}$ & & $\mathbf{N}$ & $\%$ \\
\hline CJ & - & - & 8 & - & - & - & 4 & 33 \\
\hline GL & - & - & 9 & - & - & 1 & 2 & 17 \\
\hline PF & - & - & - & 8 & 3 & 1 & 0 & 0 \\
\hline $\mathbf{C J}+\mathbf{G L}$ & - & - & 10 & - & - & - & 2 & \\
\hline $\mathbf{S P}+\mathbf{G L}$ & - & - & 1 & 2 & 3 & - & 6 & 50 \\
\hline
\end{tabular}

${ }^{\mathrm{a}}$ Zirconia catastrophic failure or zirconia cohesive failures were not considered as PTF failure. 


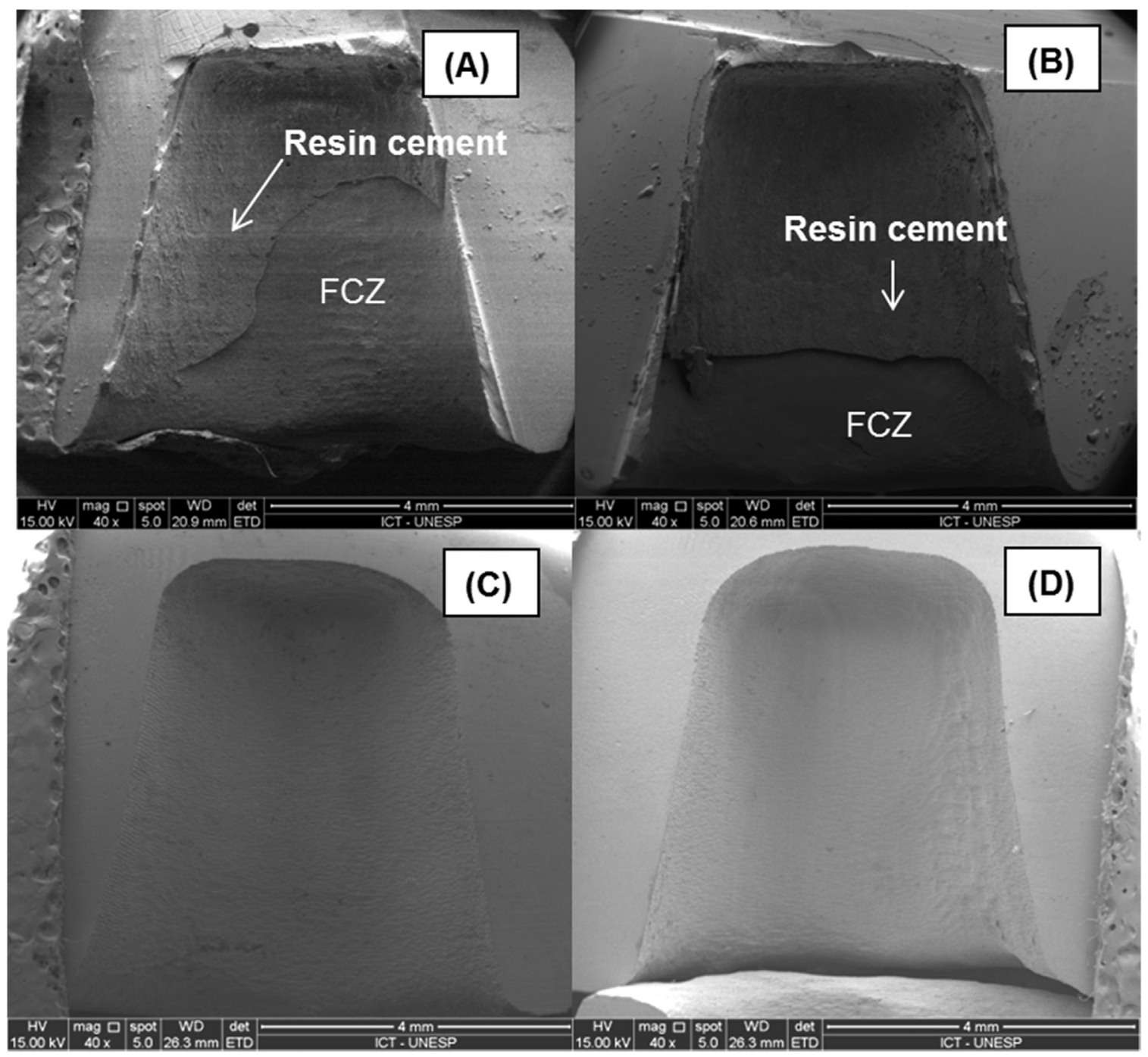

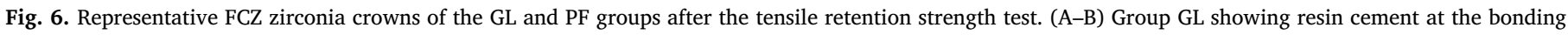
interface of the FCZ crown representing a mixed failure. (C-D) Group PF showing adhesive failure.

predictable retention/adhesion to resin cement [17]. The aging protocol (TC + storage) used in this study revealed the effects of the different bond strategies on long-term bonding. Among the different combinations, PS + GL appeared to be more sensitive to degradation when compared to the others.

The failure analysis showed that groups CJ, GL, and CJ + GL presented higher numbers of mixed failures and a higher amount of resin cement on FCZ. This indicates that the zirconia surface treatment improved resin bonding. In contrast, the PF group showed higher amounts of resin cement in the G10 substrate. One potential explanation for this could be that the G10 substrate was treated with HF and, in that particular situation (PF group), FCZ received no surface treatment. The untreated zirconia surface was unable to interlock with the resin cement, thus lowering the amount of resin cement attached to the untreated surface.

The evaluated FCZ zirconia presented a high density, homogeneous microstructure, and mechanical properties comparable to clinically available zirconia. The FCZ-glaze powder presented an XRD pattern characteristic of an amorphous material with several peaks of leucite. The use of the MDP-containing resin cement or the FCZ-inner surface treatment with glaze application could represent good strategies for working with full-contour zirconia crowns. It is important to note that a limitation of the present study was the high numbers of pretesting failures. Future studies using dentin as a substrate should be conducted to clinically validate the present findings. In addition, the tensile retention test provided information about retention between the crown and the die after different treatments; however, as an in vitro study, there are still limitations when compared to a crown submitted to the oral environment challenges.

\section{Conclusions}

The use of an MDP-containing resin cement or glaze application on the inner surface of full-contour zirconia crown has the potential to improve retention.

\section{Acknowledgments}

The first author (SAF) acknowledges funding support (2012/029450) from the São Paulo Research Foundation (FAPESP, Brazil) and Coordination for the Improvement of Higher Education Personal (CAPES/Brazil) (18922/12-0). All authors thank Drs. Tom Hill and Shashikant Singhal for their support in manufacturing FCZ crowns, Dr. Ivan Balducci for his assistance with statistical analyses, and Dr. Tais Paradella for her help with the SEM analysis. 


\section{References}

[1] Piconi C, Maccauro G. Zirconia as a ceramic biomaterial. Biomaterials 1999;20:1-25.

[2] Lazar DR, Bottino MC, Ozcan M, Valandro LF, Amaral R, Ussui V, et al. Y-TZP ceramic processing from coprecipitated powders: a comparative study with three commercial dental ceramics. Dent Mater 2008;24:1676-85.

[3] Scherrer SS, Cattani-Lorente M, Vittecoq E, de Mestral F, Griggs JA, Wiskott HW. Fatigue behavior in water of Y-TZP zirconia ceramics after abrasion with $30 \mu \mathrm{m}$ silica-coated alumina particles. Dent Mater 2011;27:28-42.

[4] Sailer I, Fehér A, Filser F, Gauckler LJ, Lüthy H, Hämmerle CH. Five-year clinical results of zirconia frameworks for posterior fixed partial dentures. Int J Prosthodont 2007;20:383-8.

[5] Roediger M, Gersdorff N, Huels A, Rinke S. Prospective evaluation of zirconia posterior fixed partial dentures: four-year clinical results. Int J Prosthodont 2010;23:141-8.

[6] Schmitt J, Goellner M, Lohbauer U, Wichmann M, Reich S. Zirconia posterior fixed partial dentures: 5-year clinical results of a prospective clinical trial. Int $\mathrm{J}$ Prosthodont 2012;25:585-9.

[7] Tinschert J, Schulze KA, Natt G, Latzke P, Heussen N, Spiekermann H. Clinical behavior of zirconia-based fixed partial dentures made of DC-Zirkon: 3-year results. Int J Prosthodont 2008;21:217-22.

[8] Guess PC, Kulis A, Witkowski S, Wolkewitz M, Zhang Y, Strub JR. Shear bond strengths between different zirconia cores and veneering ceramics and their susceptibility to thermocycling. Dent Mater 2008;24:1556-67.

[9] Sabrah AH, Cook NB, Luangruangrong P, Hara AT, Bottino MC. Full-contour Y-TZP ceramic surface roughness effect on synthetic hydroxyapatite wear. Dent Mater 2013;29:666-73.

[10] Luangruangrong P, Cook NB, Sabrah AH, Hara AT, Bottino MC. Influence of fullcontour zirconia surface roughness on wear of glass-ceramics. J Prosthodont 2014:23:198-205.

[11] Amaral R, Ozcan M, Valandro LF, Balducci I, Bottino MA. Effect of conditioning methods on the microtensile bond strength of phosphate monomer-based cement on zirconia ceramic in dry and aged conditions. J Biomed Mater Res B Appl Biomater 2008;85:1-9.

[12] Wolfart M, Lehmann F, Wolfart S, Kern M. Durability of the resin bond strength to zirconia ceramic after using different surface conditioning methods. Dent Mater 2007;23:45-50.

[13] Ntala P, Chen X, Niggli J, Cattell M. Development and testing of multi-phase glazes for adhesive bonding to zirconia substrates. J Dent 2010;38:773-81.
[14] Valentino TA, Borges GA, Borges LH, Platt JA, Correr-Sobrinho L. Influence of glazed zirconia on dual-cure luting agent bond strength. Oper Dent 2012;37:181-7.

[15] Cura C, Özcan M, Isik G, Saracoglu A. Comparison of alternative adhesive cementation concepts for zirconia ceramic: glaze layer vs zirconia primer. J Adhes Dent 2012;14:75-82.

[16] Bottino MA, Bergoli C, Lima EG, Marocho SM, Souza RO, Valandro LF. Bonding of Y-TZP to dentin: effects of Y-TZP surface conditioning, resin cement type, and aging. Oper Dent 2014;39:291-300.

[17] Lohbauer U, Zipperle M, Rischka K, Petschelt A, Müller FA. Hydroxylation of dental zirconia surfaces: characterization and bonding potential. J Biomed Mater Res B Appl Biomater 2008;87:461-7.

[18] Amaral R, Rippe M, Oliveira BG, Cesar PF, Bottino MA, Valandro LF. Evaluation of tensile retention of Y-TZP crowns after long-term aging: effect of the core substrate and crown surface conditioning. Oper Dent 2014;39:619-26.

[19] Rippe MP, Amaral R, Oliveira FS, Cesar PF, Scotti R, Valandro LF, et al. Evaluation of tensile retention of Y-TZP crowns cemented on resin composite cores: effect of the cement and Y-TZP surface conditioning. Oper Dent 2015;40:1-10.

[20] Iost A, Bigot R. Indentation size effect: reality or artefact? J Mater Sci 1996;31:3573-7.

[21] Ponton $\mathrm{CB}$, Rawlings RD. Vickers indentation fracture-toughness test part 1 review of literature and formulation of standardized indentation toughness equations. Mater Sci Technol 1989;5:865-72.

[22] Ponton CB, Rawlings RD. Vickers indentation fracture-toughness test part 2 application and critical-evaluation of standardized indentation toughness equations Mater Sci Technol 1989;5:961-76.

[23] Corazza PH, Feitosa SA, Borges AL, Della Bona A. Influence of convergence angle of tooth preparation on the fracture resistance of Y-TZP-based all-ceramic restorations. Dent Mater 2013;29:339-47.

[24] Ernst CP, Cohnen U, Stender E, Willershausen B. In vitro retentive strength of zirconium oxide ceramic crowns using different luting agents. J Prosthet Dent 2005;93:551-8.

[25] Lüthy H, Loeffel O, Hammerle CH. Effect of thermocycling on bond strength of luting cements to zirconia ceramic. Dent Mater 2006;22:195-200.

[26] Garcia Fonseca R, de Oliveira Abi-Rached F, dos Santos Nunes Reis JM, Rambaldi F, Baldissara P. Effect of particle size on the flexural strength and phase transformation of an airborne-particle abraded yttria-stabilized tetragonal zirconia polycrystal ceramic. J Prosthet Dent 2013;110:510-4.

[27] Feitosa SA, Lima NB, Yoshito WK, Campos F, Bottino MA, Valandro LF, et al. Bonding strategies to full-contour zirconia: zirconia pretreatment with piranha solution, glaze and airborne-particle abrasion. Int J Adhes Adhes 2017;77:151-6. 